\title{
Editorial
}

\section{Dynamics and Control in Sciences and Engineering}

J. M. Balthazar

Received 19 August 2007; Accepted 19 August 2007

Copyright ( $) 2007$ J. M. Balthazar. This is an open access article distributed under the Creative Commons Attribution License, which permits unrestricted use, distribution, and reproduction in any medium, provided the original work is properly cited.

The aim of this special issue is to present discussions of some recent developments in the area of dynamics and control, in all branches of science and engineering.

The subject of dynamics and control systems is wonderfully broad and it has important applications in fields ranging from several engineering branches, physics, and computer science to the life sciences, sociology, and finance.

So, this special issue of MPE is designed to present the state-of-the-art research and the latest theoretical, numerical, and practical achievements to contribute to the advancement of this field, in a significant way.

This special issue involves 19 original papers, selected by the editor, related to the various researches themes on dynamics and control, in order to present recent results on the mentioned fields.

These papers are related to various subjects: mechanical (modeling, dynamics, robotics, structures, chaos); electrical (telecommunications), aerospace science, biological (modeling), fluids and control processes (optimization).

This special issue on Dynamics and Control in Sciences and Engineering papers is organized as follows:

Concerning chaos, the 4 papers are:

Successive Bifurcation Conditions of a Lorenz-Type Equation for the Fluid Convection Due to the Transient Thermal Field, Xiaoling He. 
Patrol Mobile Robots and Chaotic Trajectories, Luiz S. Martins-Filho and Elbert E. N. Macau.

Inductorless Chua's Circuit: Experimental Time Series Analysis, R. M. Rubinger, A. W. M. Nascimento, L. F. Mello, C. P. L. Rubinger, N. Manzanares Filho, and H. A. Albuquerque. Chaos Synchronization Criteria and Costs of Sinusoidally Coupled Horizontal Platform Systems, Jianping Cai, Xiaofeng Wu, and Shuhui Chen.

\section{Concerning control and identification, the 6 papers are:}

Stabilizability and Motion Tracking Conditions for Mechanical Nonholonomic Control Systems, Elżbieta Jarzębowska.

Stabilization and Observability of a Rotating Timoshenko Beam Model, Alexander Zuyev and Oliver Sawodny.

Numerical and Analytical Study of Optimal Low-Thrust Limited-Power Transfers between Close Circular Coplanar Orbits, Sandro da Silva Fernandes and Wander Almodovar Golfetto.

Fault Detection and Control of Process Systems, Vu Trieu Minh, Nitin Afzulpurkar, and W. M. Wan Muhamad.

Joint Dynamics Modeling and Parameter Identification for Space Robot Applications, Adenilson R. da Silva, Luiz C. Gadelha de Souza, and Bernd Schäfer.

Quadratic Stabilization of LPV System by an LTI Controller Based on ILMI Algorithm, Wei Xie.

Concerning dynamics, the 3 papers are:

Modal Formulation of Segmented Euler-Bernoulli Beams, Rosemaira Dalcin Copetti, Julio C. R. Claeyssen, and Teresa Tsukazan.

Asymptotic Solution of the Theory of Shells Boundary Value Problem, I. V. Andrianov and J. Awrejcewicz.

Dynamic Stationary Response of Reinforced Plates by the Boundary Element Method, Luiz Carlos Facundo Sanches, Euclides Mesquita, Renato Pavanello, and Leandro Palermo Jr.

Concerning dynamics and control applications, the 2 papers are:

Simple Orbit Determination Using GPS Based on a Least-Squares Algorithm Employing Sequential Givens Rotations, Rodolpho Vilhena de Moraes, Aurea Aparecida da Silva, and Helio Koiti Kuga.

Evaluation of Tropospheric and Ionospheric Effects on the Geographic Localization of Data Collection Platforms, C. C. Celestino, C. T. Sousa, W. Yamaguti, and H. K. Kuga. 
Concerning turbulence, the 2 papers are:

Incompressible Turbulent Flow Simulation Using the $\kappa-\varepsilon$ Model and Upwind Schemes, V. G. Ferreira, A. C. Brandi, F. A. Kurokawa, P. Seleghim Jr., A. Castelo, and J. A. Cuminato. Dynamical Simulation and Statistical Analysis of Velocity Fluctuations of a Turbulent Flow behind a Cube, T. F. Oliveira, R. B. Miserda, and F. R. Cunha.

Concerning Biological applications, the paper is:

A Stochastic Model for the HIV/AIDS Dynamic Evolution, Giuseppe Di Biase, Guglielmo D’Amico, Arturo Di Girolamo, Jacques Janssen, Stefano Iacobelli, Nicola Tinari, and Raimondo Manca.

Concerning telecommunications, the paper is:

Models for Master-Slave Clock Distribution Networks with Third- Order Phase-Locked Loops, José Roberto Castilho Piqueira and Marcela de Carvalho Freschi.

J. M. Balthazar: Department of Statistics, Applied Mathematics and Computation, State University of São Paulo at Rio Claro, P.O. Box 178, 13500-230 Rio Claro, SP, Brazil Email address: jmbaltha@rc.unesp.br 


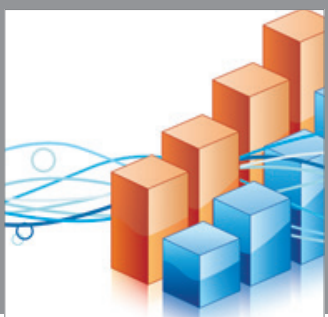

Advances in

Operations Research

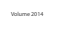

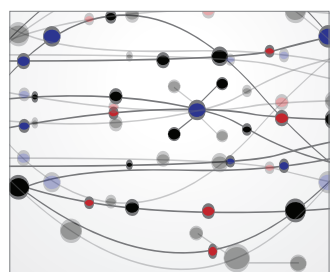

\section{The Scientific} World Journal
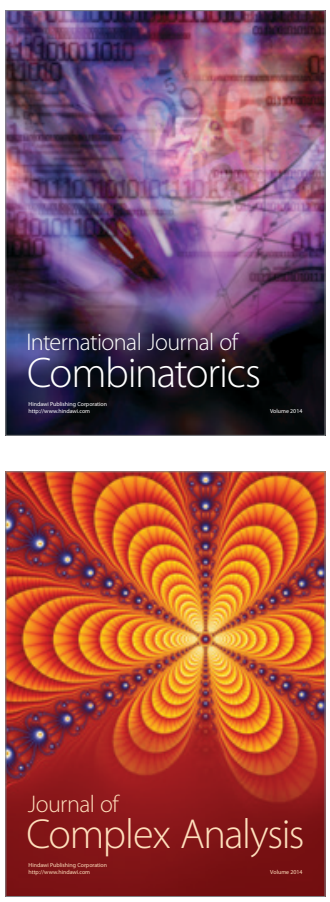

International Journal of

Mathematics and

Mathematical

Sciences
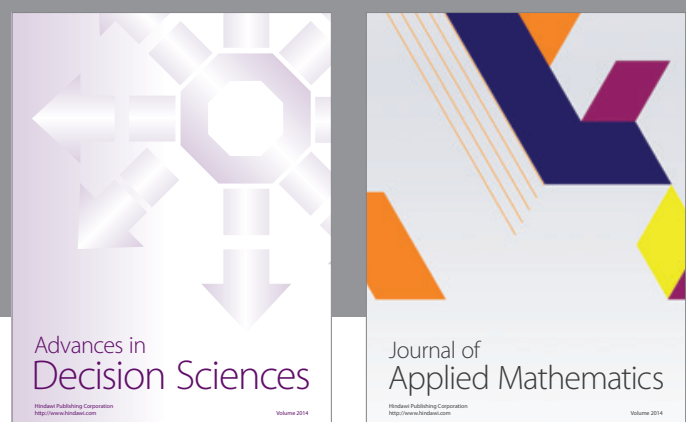

Journal of

Applied Mathematics
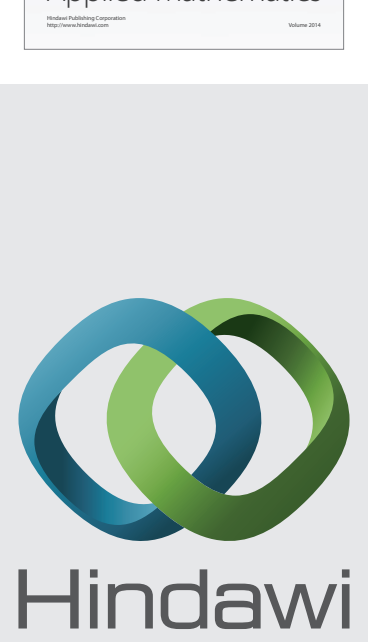

Submit your manuscripts at http://www.hindawi.com
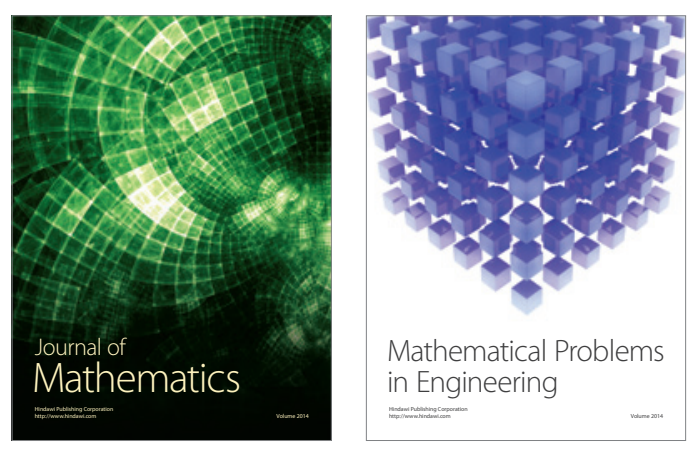

Mathematical Problems in Engineering
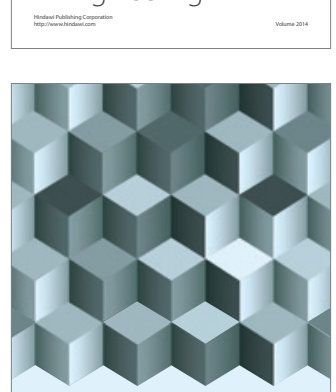

Journal of

Function Spaces
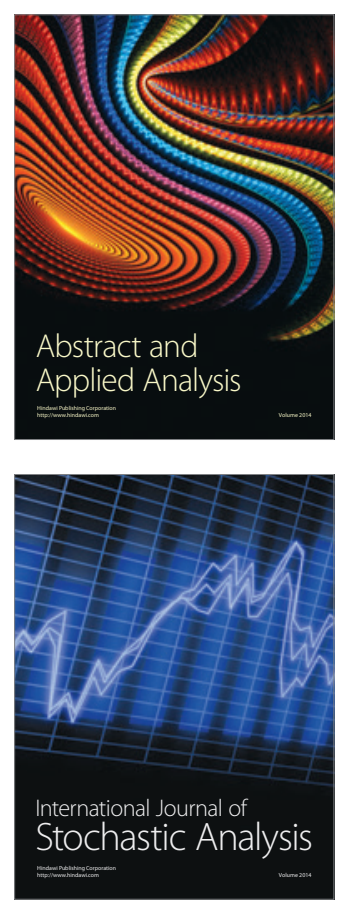

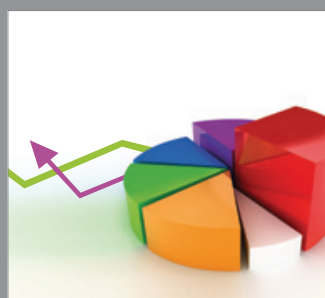

ournal of

Probability and Statistics

Promensencen
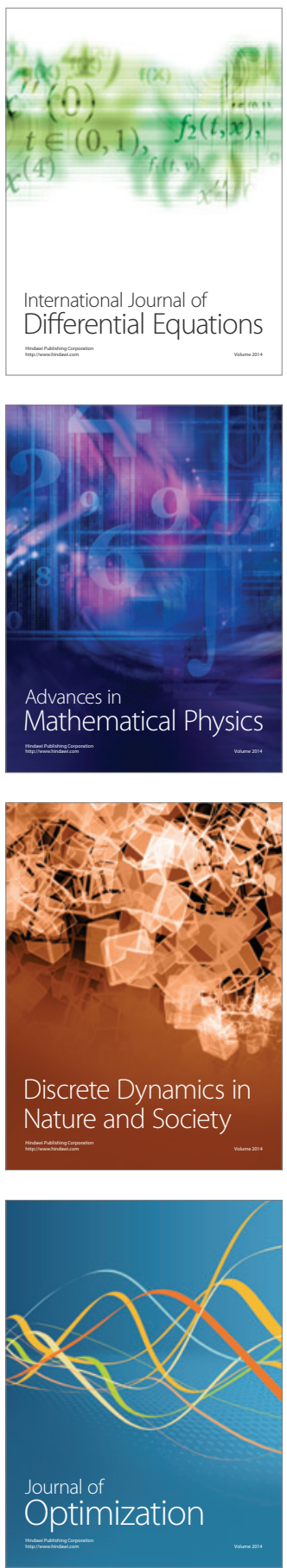